\title{
Characteristics and Application Analysis of Proprietary Chinese Medicine Containing Chrysanthemum
}

\author{
Zhen-zhen $\mathrm{WEI}^{1, \mathrm{a}}$, Xiao-yan $\mathrm{FANG}^{2, \mathrm{~b}}$, Ming BAI ${ }^{3}$ and Ming-san \\ MIAO ${ }^{4, c, *}$ \\ ${ }^{1,2}$ Hennan University of Chinese Medicine,Zhengzhou,China 450008 \\ aweizhenzhen08@126.com, ${ }^{\mathrm{b}}$ fxylele@yeah.net, ${ }^{\mathrm{c}}$ miaomingsan@163.com \\ ${ }^{*}$ Corresponding author
}

Keywords: Traditional Chinese medicine chrysanthemum, Proprietary Chinese medicine, Efficacy, Clinical application

\begin{abstract}
Purposes: To sum up the characteristics of Chinese medicine with chrysanthemum and lay a foundation for the development of new dosage forms and the rational application of Chinese Medicine. Methods: According to the Chinese Pharmacopoeia contains chrysanthemum in the pharmaceutical dosage form, drug composition, efficacy, matters needing attention and so on carries on the analysis summary. The author sorted out the journal literature about proprietary Chinese medicine containing Chrysanthemum in the China National Knowledge Infrastructure (CNKI), and analyzed its application in clinical practice. Results: The proprietary Chinese medicine containing chrysanthemum was widely used in the treatment of various diseases in clinic, but it was taken orally and without any external use of Chinese patent medicine. Moreover, the medicine composition of some Chinese medicine containing chrysanthemum was complicated, so it's necessary to clarify its pharmacological action. Conclusions: The development of new Chinese medicine for external use, clear its pharmacological action, reduce the composition of drugs, avoid the occurrence of adverse reactions, the Chinese medicine contains chrysanthemum will have a broad prospect.
\end{abstract}

\section{Introduction}

Chrysanthemum is a perennial herbaceous plant. The medicinal properties of its agents, including sweet, bitter, slightly cold. Return to the lung and liver meridian. With the effect of calming the liver and improving eyesight, clearing away heat and detoxification $[1,2]$. As an important medicinal plant, its chemical composition is complex and widely used in food, tea and pharmaceutical products. Modern use the new research methods have been isolated from the chrysanthemum flavonoids, terpenoids and organic acids and other chemical composition. With anti-tumor, blood lipid, antibacterial, analgesic, immune regulation, anti-fatigue and other pharmacological effects [3]. This article through the 2015 version of Chinese Pharmacopoeia contains chrysanthemum proprietary Chinese medicine according to its dosage form, drug composition, precautions, the use of taboo, and clinical application of systematic summary and analysis. Then, we sorted out the China National Knowledge Infrastructure (CNKI) Periodicals about Chinese patent medicine, and analyzed its application in clinical practice, laid the foundation for the rational application in the future, and promoted the innovation and development of chrysanthemum proprietary Chinese medicine in the field of Chinese medicine. 


\section{Chinese Pharmacopoeia Contains Chrysanthemum Proprietary Chinese Medicine Present Situation and Analysis}

2015 version of Chinese Pharmacopoeia contains a total of 68 kinds of Chinese medicine chrysanthemum. The dosage forms are various, including tablets, pills, capsules, granules, oral liquid and so on, see Table 1 for details. Chinese Pharmacopoeia contains chrysanthemum proprietary Chinese medicine from the lowest single herbs composition (wild chrysanthemum suppository), the highest composition by the 28 herbs (compound Yigan pill), it can be divided into five sections of herbs, namely: 1-5 flavors 5 kinds, 6-10 flavors 19 species, 11-15 flavors 11 kinds, 16-20 flavors 23 kinds and> 20 flavors 10 kinds.

Table 1 Distribution of dosage forms of proprietary Chinese medicine containing Chrysanthemum

\begin{tabular}{ccccccc}
\hline Dosage form & tablets & pills & capsule & granules & $\begin{array}{c}\text { Liquid } \\
\text { preparation }\end{array}$ & others \\
\hline quantity & 16 & 25 & 11 & 8 & 6 & 2 \\
Percentage $(\%)$ & 23.5 & 36.8 & 16.2 & 11.8 & 8.8 & 2.9 \\
\hline
\end{tabular}

Of the 68 varieties of chrysanthemum in Chinese pharmacopoeia, only 5 of them are composed of 1-5, accounting for $7 \%$ of the total. The drugs are not the more the better, because drugs pull each other and will deviate from the original function of the site. Therefore, with the increase of flavors in the number, the effect of Chrysanthemum in Chinese medicine will be weakened, so we should use modern research techniques combined with the theoretical knowledge of traditional Chinese medicine to develop proprietary Chinese medicine based on chrysanthemum. In addition, chrysanthemum and the effect of the same drugs co existed in the same medicine and played the same effect in some Chinese medicines. Whether we can choose a replacement for the effect of the same medicinal materials, increase its dosage, its feasibility for further study.

\section{Chinese Pharmacopoeia Contains Chrysanthemum Proprietary Chinese Medicine Efficacy Classification and Analysis}

2015 version of Chinese Pharmacopoeia have 68 kinds of Chinese medicine contains chrysanthemum. According to its functional indications, it can be divided into four categories. Each of the representatives of Chinese medicine and the number see Table 2. In these 68 kinds of proprietary Chinese medicines, wild chrysanthemum 15 kinds of medicines, the rest are chrysanthemum medicines. Wild chrysanthemum, sexual bitter, to the liver and heart, the main effect is clearing away heat and toxic material, and its power is better than chrysanthemum; To the wild chrysanthemum medicine proprietary Chinese medicines are clearing away heat and toxic material, therefore, the medicines will be classified as clearing away heat and toxic material, dispelling wind and relieve sore throat. 
Table 2 Efficacy classification, drug representation and number of proprietary Chinese medicine containing Chrysanthemum

\begin{tabular}{|c|c|c|}
\hline Efficacy & Drug representation & Number \\
\hline $\begin{array}{l}\text { Clearing away heat and toxic material, } \\
\text { dispelling wind and relieve sore throat }\end{array}$ & $\begin{array}{l}\text { Xiaoer Ganmao granules,Huanglian } \\
\text { Shangqing tablets }\end{array}$ & 39 \\
\hline $\begin{array}{l}\text { Tonifying liver and kidney, removing } \\
\text { nebula }\end{array}$ & $\begin{array}{l}\text { Qiju Dihuang pills, } \\
\text { Mingmu Dihuang pills }\end{array}$ & 16 \\
\hline $\begin{array}{l}\text { Nourishing the blood and soothe the } \\
\text { nerves, strengthen the body and promote the } \\
\text { brain }\end{array}$ & $\begin{array}{c}\text { Kunbao pills, } \\
\text { Shenwu Jiannao Capsules }\end{array}$ & 5 \\
\hline $\begin{array}{l}\text { Calming the liver to stop the } \\
\text { wind,activating blood circulation to } \\
\text { dissipate blood stasis }\end{array}$ & $\begin{array}{c}\text { Naomaitai Capsules, } \\
\text { Shuanghu Qinggan granules }\end{array}$ & 8 \\
\hline
\end{tabular}

In the above Chinese medicines, the wild chrysanthemum bolt is the anal drug, which belongs to the external use, and the rest of the Chinese medicines are taken orally. Therefore, the development of chrysanthemum is used as an intermediate medicine, which can be a direction of the study of chrysanthemum chrysanthemum in the future.

\section{Chrysanthemum in the Application of Proprietary Chinese Medicine Form}

Chrysanthemum can be taken orally: decoction, $10-15 \mathrm{~g}$ or into the pill, scattered or tea. Topical: amount, pound or decoction wash. Chinese Pharmacopoeia contains 68 kinds of chrysanthemum with Chinese medicines, chrysanthemum medicines were water decoction, distillation extraction of volatile oil, research into fine powder, water or ethanol hot dip, no external pound or decoction of Chinese medicine, as shown in table 3.

Table 3 Application forms of Chrysanthemum in preparation of proprietary Chinese medicine

\begin{tabular}{ccccc}
\hline Medicinal form & Decoction & Volatile oil & Fine powder & Hot dip \\
\hline Number & 27 & 12 & 21 & 8 \\
Percentage $(\%)$ & 39.7 & 17.6 & 30.9 & 11.8 \\
\hline
\end{tabular}

\section{Chinese Pharmacopoeia Contains Chrysanthemum Proprietary Chinese Medicine Note}

Wild chrysanthemum bitter cold, long-term use or dosage is too large, will hurt the spleen and stomach yang, stomach discomfort, poor appetite, bowel, loose stools and other adverse reactions. Spleen and stomach and pregnant women are not appropriate. Pharmacopoeia contains chrysanthemum proprietary Chinese medicine; there are 25 kinds of notes. Among them, medicines should pay attention to the diet during the 8 species, elderly, pregnant women, children and lactating women with caution 12 
species, 5 kinds of spleen and stomach deficiency, 3 of other diseases. Besides there are 2 adverse reactions during drug administration, as shown in table 4. Because a drug may have multiple points of attention, there is a cross-phenomenon when classifying, and there will be more than 25 kinds of drugs in the total number of drugs, the percentage of which is the proportion of all Chinese medicine.

Table 4 Distribution of notes of proprietary Chinese medicine containing Chrysanthemum

\begin{tabular}{cccccc}
\hline Notes & $\begin{array}{c}\text { Pay } \\
\text { attention to } \\
\text { diet }\end{array}$ & $\begin{array}{c}\text { Children with } \\
\text { pregnant women } \\
\text { with caution }\end{array}$ & $\begin{array}{c}\text { Spleen and } \\
\text { stomach Deficiency } \\
\text { with caution }\end{array}$ & Others & $\begin{array}{c}\text { Advers } \\
\text { e reactions }\end{array}$ \\
\hline Number & 8 & 12 & 5 & 3 & 2 \\
Percentage & 11.8 & 17.7 & 7.4 & 4.4 & 2.9 \\
\hline
\end{tabular}

\section{The Clinical Application and Adverse Reactions of Proprietary Chinese Medicine Containing Chrysanthemum Included in CNKI}

\section{Analysis of Clinical Application of Chrysanthemum Proprietary Chinese Medicines}

Clearing Away Heat and Detoxification. Chinese Pharmacopoeia records chrysanthemum with the effect of clearing away heat and toxic material, In clinical it were used for the treatment of cold, upper respiratory tract infection, fever, pharyngitis, headache and so on. Children due to the development of the body system is not mature, it's vulnerable to external bacterial infection and then a cold. Xiaoer Ganmao granules is a new kind of compound preparations, its heat-clearing and detoxifying effect is stronger, it can be used for exogenous wind hot, cough, fever, headache, etc. A study of 100 cases of acute upper respiratory tract infection in rheumatoid children, the clinical use of pediatric cold particles combined with traditional Chinese medicine bathing treatment, the results can significantly shorten the course of children and fever time [4, 5]. Laboratory studies have shown that Xiaoer Ganmao granules in mice caused by chemical stimulation of cough, cough can extend the mouse incubation period; it can reduce the number of cough, and enhance their ability to resist evil [6]. According to the pathological characteristics of Xiaocuo pill to acne, Clinical use of acne pills combined with red and blue light treatment of mild to moderate acne total effective rate of $85.41 \%$, safely and effectively [7].

Eliminate nebula and brighten the eyes. Compendium of Materia Medica records chrysanthemum "wind fever, the eye ache wants to take off, the tear goes out, raise the eye to blind, be the pillow can improve eyesight". Cataract is caused by essence and blood deficiency and stagnated heat of liver channel. Fuming Tablets can effectively relieve cataract by tonifying liver and kidney and can accelerate the eye nutrition metabolism, improve eyes blood circulation ability, effectively improve the lens of the turbidity phenomenon [8,9]; Diabetic retinopathy patients with vascular permeability enhancement, blood - aqueous barrier difference, clinical oral Fuming Tablets treatment of cataract patients with diabetic retinopathy, can expand peripheral blood vessels, reduce vascular permeability, promote the vision recovery of patients, and can 
reduce postoperative inflammatory response[10]. Mingmu Dihuang pill can nourish the liver and kidney, improve eyesight. 60 patients with optic atrophy were randomly divided into two groups. The control group was injected compound Anisodine injection. The observation group was given Mingmu Dihuang pills on the basis of control group. The results showed that the application of Mingmu Dihuang pills combined with compound Anisodine injection in the treatment of optic atrophy can improve the static and dynamic visual field and improve the eyesight [11].

Purging Fire and Relieve Pain. Niuhuang Shangqing capsules can clear heat and purge fire, dispel wind and relieve pain. Clinical use has a better curative effect on Niuhuang Shangqing capsules in treatment of gingivitis and fire. It can significantly improve the patient's upset, insomnia, more dreams and other accompanying symptoms [12]. Huanglian Shangqing pills have clearing heat and purging fire, dispelling wind analgesic efficacy. It combined with carbamazepine not only can prolong drug effective time and also can reduce the recurrence of the disease, relieve pain for patients suffering from trigeminal neuralgia [13].

Other. Contains chrysanthemum proprietary Chinese medicines also has the function of activating blood stasis, supplementing qi and nourishing blood, etc. It can also treat cerebral infarction, vascular dementia, hypertension, insomnia, dizziness and other diseases. For example, Shenwu Jiannao capsules consist of 19 Chinese herbs, including ginseng, chrysanthemum and scutellaria, which are used to supplement the kidney, nourish the blood and strengthen the brain. Modern pharmacological research shows that this product has the function of anti - aging, improving cerebral circulation, enhancing cerebral blood flow, improving brain function, can improve the patients with insomnia symptoms, and insomnia patients with depression and anxiety have a certain improvement [14]. Naomaitai capsules are commonly used in clinical treatment of ischemic diseases. It can combine with Oxiracetam Injection for the treatment of vascular dementia has better clinical efficacy, it can improve cognitive function and quality of life, reduce the level of inflammatory factor [15].

\section{Adverse Reaction Analysis}

In the CNKI with chrysanthemum proprietary Chinese medicine, some drugs will lead to the occurrence of adverse reactions. For example, Niuhuang Shangqing capsules, some patients suffered from chest tightness, dizziness, nausea and facial edema after taking the medicine, followed by difficulty in breathing, cold sweats and unconsciousness; After have patients take on Niuhuang Shangqing pills for clearing the fever, and shortness of breath, itchy skin. After secondary treatment, the symptoms happened again. Excluding other factors, confirmed that the Niuhuang Shangqing pills caused by allergy, but its composition is complex, the sensitizing components are difficult to determine; In the past health patients, Hemolytic anemia appeared after taking Niuhuang Shangqing tablets a lot. According to Chinese medicine dictionary, may be caused by its main ingredients bezoar dosage is too large, but the exact cause remains to be further discussed.

Chinese medicine is a kind of traditional Chinese medicine, which is made from Chinese herbal medicine and processed into different dosage forms. it contains many kinds of Chinese herbal medicine. Adverse reactions occurred in the clinical application of proprietary Chinese medicine containing chrysanthemum, not necessarily the problem of the chrysanthemum itself. There are 10 kinds of proprietary Chinese medicines containing chrysanthemum in Chinese Pharmacopoeia. They are made up of twenty or more Chinese herbs. Serious adverse reactions may also occur 
when processed into proprietary Chinese medicines. Therefore, the Chinese traditional medicine ingredients containing chrysanthemum complex; The pharmacological and toxicological effects are not clear, so whether the adverse reaction of Chinese medicine containing chrysanthemum is related with chrysanthemum and adverse reaction needs further study.

\section{Results}

Chrysanthemum in China has more than 3,000 years of history; The Book of Songs and Qu Yuan's Lament have chrysanthemum records. As a kind of food and edible flowers, chrysanthemum is widely used in the field of Chinese medicine. Chrysanthemum proprietary Chinese medicine can be used to treat headache dizziness, high blood pressure, cataracts, sore carbuncle swollen poison and other diseases in clinical. Compared with Western medicine, proprietary Chinese medicine is particularly unique advantages. Such as the treatment of sleep disorders and insomnia, the traditional side effects of Western medicine treatment are easy to addiction, but traditional Chinese medicine has the advantage of not becoming addicted.

Through the analysis of Chinese Pharmacopoeia contains chrysanthemum proprietary Chinese medicine and the CNKI of clinical applications, there are some problems. There are many proprietary Chinese medicines used in the amount of medication for children only Note: children reduce or follow the doctor's advice and not clear in the use and it is difficult to grasp the amount. In these 68 kinds of proprietary Chinese medicine from the 1-5 flavors composition only has 5 kinds, accounting for $7 \%$ of the total. Not the more the better herbs, with the increase in the number of flavors, chrysanthemum in the role of proprietary Chinese medicine will be weakened; Drugs that pull each other may have the opposite effect. In addition, the adverse reactions in the report were not analyzed the reasons and the safety of medicine need to be further considered.

\section{Conclusions}

In the future study of chrysanthemum should further clarify the dose of children or find their alternative drugs; Clear the efficacy of Chinese herbal medicine in the efficacy of various herbs and try to reduce the number of Chinese medicine containing chrysanthemum proprietary Chinese medicines, and choose the drugs with stronger efficacy. When the adverse reaction occurs, we should analyze the specific causes and find out the compatibility of single or multiple drugs which cause adverse reactions, so as to avoid the occurrence of adverse reactions. The value of chrysanthemum is not only in the field of medicine, but also in the field of food. Chrysanthemum tea, chrysanthemum wine, chrysanthemum soup and others have highly nutritional value. We can to further explore the value of chrysanthemum in other areas. Using modern technology to promote the development of Chinese medicine.

\section{Acknowledgement}

This research was financially supported by the the National international cooperation base (State University letter 2016-65), the Central Plains scholars (162101510003), Henan Province campus cooperation award ((2016) 38). 


\section{References}

[1] National Pharmacopoeia Commission. People's Republic of China Pharmacopoeia: 2015 ed., Beijing: China Medical Science and Technology Press, 2015. In Chinese

[2] Liu Guohua, Zhang Yanmin. J. Chinese Journal of Practical Medicine, 2012, 7 (7): 244. In Chinese

[3] Qu Lu, Wang Tao, etc. J. Drug Evaluation Research, 2015, 38 (01): 98-104. In Chinese

[4] Zhang Jie. J. Chinese Medicine Guide, 2016, 14 (25): 192-193. In Chinese

[5] Gao Fazhi. J. Clinical medicine research and practice, 2016, 1 (06): 56. In Chinese

[6] Lei Jing. J. The world's latest medical information digest, 2015, 15 (37): 92 - 113. In Chinese

[7] Wu Xiaofei, Wang Kaiyun, Xu Xia. J. Chinese Medical Abstract (Skin Science), 2015, 32 (01): 103-104. In Chinese

[8] Lwase T, Jo YJ, Oveson BC. J.BMC Ophthalmol, 2013, 13 (1) :77-79.

[9]Park H. J. Graefes Archive for Clin Experimental Ophthalmology, 2011, 249 (1) :151-152.

[10] Shao Qionghua. J. Southwest National Defense Medicine, 2016, 26 (1): 62-65. In Chinese

[11] He Rizhu. J. Chinese Journal of Medicine, 2017, 15 (05): 183-184. In Chinese

[12] Wang Huanwen. J. Fujian Traditional Chinese Medicine, 2013, 44 (05): 32-33. In Chinese

[13] Zhang Siling, Liao Jiayou. J. Laboratory Medicine and Clinic, 2014, 11 (01): 71-73. In Chinese

[14] Guo Fengyi, Shen Yulian, Guo Jianfeng. J. Chinese Journal of experimental Chinese medicine science, 2014, 20 (12): 230-233. In Chinese

[15] Wang Juan. J. Modern medicine and clinic, 2017, 32 (05): 792-795. In Chinese 